\title{
A Mixed Mode Self-Programming Neural System-on-Chip for Real-Time Applications
}

\author{
Khurram Waheed and Fathi M. Salam \\ Department of Electrical and Computer Engineering \\ Michigan State University \\ East Lansing, MI 48824-1226
}

\begin{abstract}
The paper provides an overview of the development of a self-learning computing chip in the new 0.18 micron copper technology. The chip realizes an architecture that achieves the task of self-learning execution times in the micro to milli seconds. The core consists of basic building blocks of 4quadrant multipliers, trans-conductance amplifiers, and active load resistances, for analog (forward-) network processing and learning modules. Super-imposed on the processing network are digital memory and control modules composed of D-Flip-flops, ADC, Multiplying D/A Converter (MDAC), and comparators for parameter (weight) storage, logical control and analog/digital conversions.
\end{abstract}

The single System-on-Chip design impacts several domains of critical applications that include nano-scale biotechnology, automotive sensing, control and actuation, wireless communications, image feature extraction and pattern matching etc

\section{Introduction}

The core of the chip is a neurally inspired scalable (reconfigurable) array network for compatibility with VLSI. The chip is endowed with tested auto-learning capability, realized in hardware, to achieve global task auto-learning execution times in the micro to milli seconds.

The architectural forward network (and learning modules) process in analog continuous-time mode, while the (converged, steady state) weights/parameters can be stored on chip in digital form. The overall architectural design adopts engineering methods from adaptive networks and optimization principles [1,2].

The designed chip can handle 16 inputs, 16 outputs. In addition there are inputs for control interface, synchronization and stand-alone programmability of the chip resulting in an approximate die area of $4000 \times 6000$ $\mu \mathrm{m}^{2}$ in a QFP-208L package
The 6 layer Copper $(\mathrm{Cu})$ interconnect, single poly, 0.18 micron process enables dense connectivity and dense die area of this highly interconnected network resulting in a compact powerful engine. Moreover, the special low resistance and low capacitance electrical properties of copper permit the design to achieve the high connectivity while still managing precise distributions of resistive and capacitive loads. These properties enable one to predict performance and limit signal time-delays along the interconnect. The small feature size and the electrical interconnect properties for copper are enablers to the realization of such a powerful chip with dense interconnectivity.

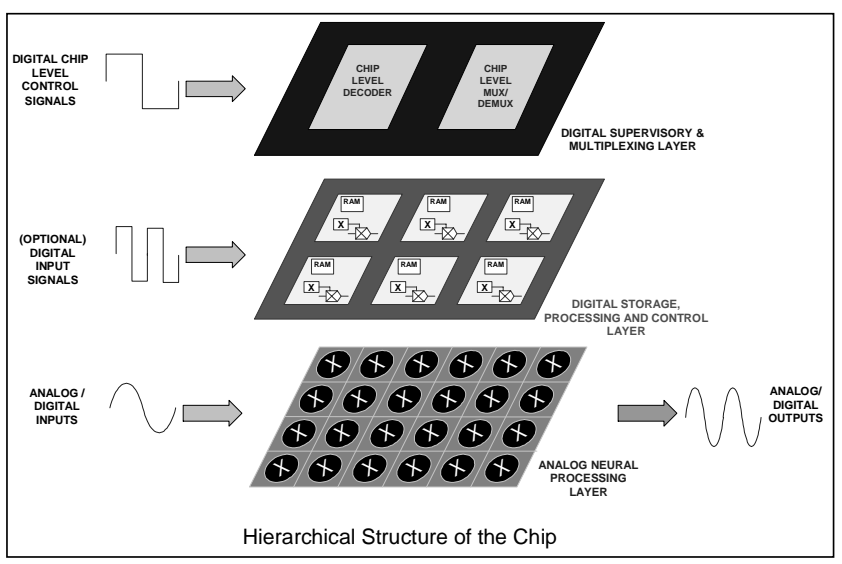

Figure 1: Arcitectural Overview of the Chip

The chip operates in four different modes: (i) learn, (ii) (on-chip) store, (iii) program read/write, and (iv) process which selectively combine its intrinsic analog and digital building blocks in a novel manner (see fig. 1).

Initially the system-level chip design was simulated and verified using SIMULINK/MATLAB. All the building blocks were custom designed and extensively simulated using HSPICE (incorporating the UMC level 49 models). The design was implemented in the 6 level Copper $(\mathrm{Cu})$ interconnect, single poly, 0.18 micron process which is an enabler for the dense connectivity and dense die area of this highly interconnected network. The design was laid out and verified using Cadence Tools. More details of 
high-level design, circuit design of major blocks and chiplayout will be provided in the subsequent sections $[8,9]$.

The resulting chip design requires no traditional programming or coding [2,3]. In addition to novel architectural design, the hardware also performs the heavy computational burden by selectively realizing programmability as on-chip auto-learning modules. The resulting System-on-Chip operates on $1.5 \mathrm{~V}$ power source and consumes approximately $1 \mathrm{mWatt}$ of power.

\section{Architectural Design}

The design process was comprised of consecutive stages, based on a top-down definition of the chip. A general definition of the functionality and intended applications was created, and the development of the chip design was done in three different levels.

- A high-level design, specifying the characteristics of the neural network to be implemented and the definition of its basic building blocks.

- A circuit level design, describing each of these blocks based on the copper technology with their corresponding simulations.

- Finally a layout level design, where the actual chip layout is created and verified

\section{High Level Design: Modified BP Algorithm}

We present our stepwise approach for tailoring the BP algorithm so that it becomes suitable for VLSI implementation. For illustration in each case, we present the simulated results of the XOR problem, highlighting the effect of each modification on the performance of the algorithm.

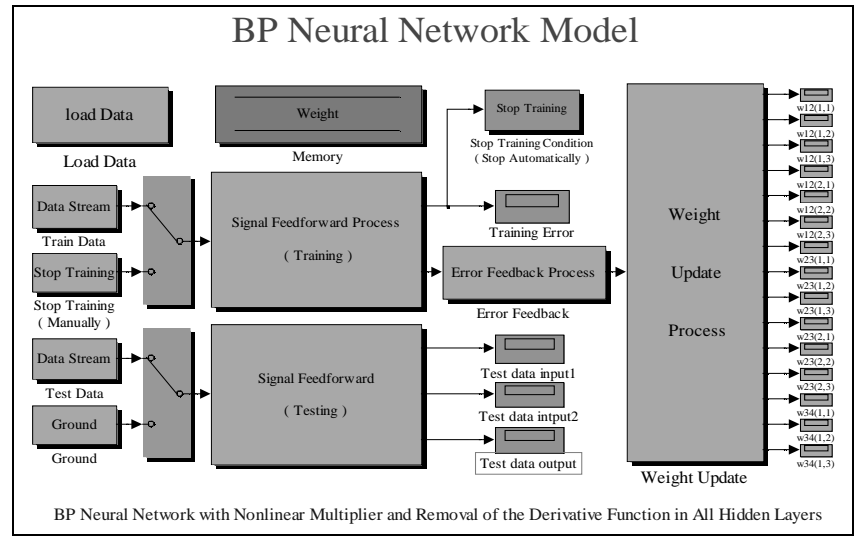

Figure 2: Simulink Model for Chip Simulations

For our simulations, we constructed a four-layer neural network, it has an input layer, two hidden layers and an output layer. The nonlinear mapping function is used only by the neurons in the two hidden layers. For the XOR simulation we are illustrating, there are two input neurons and one output neuron.

The update law in each case is derived from the mathematical model for a multi-layer feed-forward neural network.. In the equations below

$\dot{W}^{(3)}$ - represents the time derivative of the weight for output layer

$\dot{W}^{(2)}$ - represents the time derivative of the weight for $2^{\text {nd }}$ hidden layer

$\dot{W}^{(1)}$ - represents the time derivative of the weight for $1^{\text {st }}$ hidden layer

\subsection{Modified BP algorithm with linear multiplier.}

Using the weight update rule

$\dot{W}=-\eta \cdot \frac{\partial E}{\partial W}-\alpha \cdot W$ where $\quad N E T^{(i)}=W^{(i)} \cdot X^{(i-1)}$

The update laws are

$$
\begin{aligned}
\dot{W}^{(3)}= & \eta \cdot(D-Y) \cdot X^{(3)}-\alpha \cdot W^{(3)} \\
\dot{W}^{(2)}= & \eta \cdot \psi^{\prime}\left(N E T^{(2)}\right) \cdot\left((D-Y) W^{(3)}\right) \cdot X^{(2)}-\alpha \cdot W^{(2)} \\
\dot{W}^{(1)}= & \eta \cdot \psi^{\prime}\left(N E T^{(1)}\right) \cdot\left(\psi^{\prime}\left(N E T^{(2)}\right) \cdot\right. \\
& \left.\left((D-Y) \cdot W^{(3)}\right) \cdot W^{(2)}\right) \cdot X^{(1)}-\alpha \cdot W^{(1)}
\end{aligned}
$$
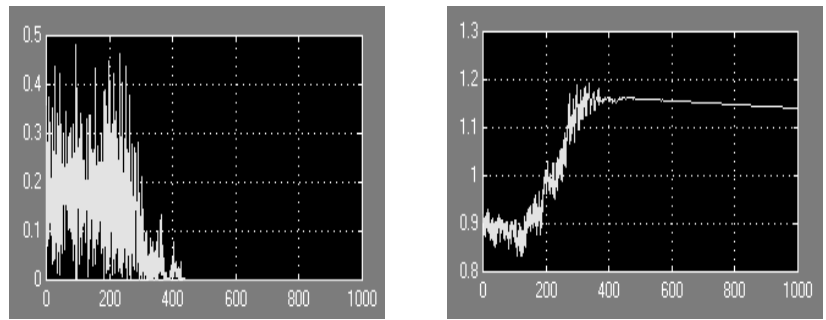

Figure 3: Training Error and convergence for the modified BP Algorithm

\subsection{Modified BP algorithm with nonlinear multiplier.}

This update law and the subsequent law considers the effect of the presence of resistive elements in hardware circuits and the multiplication using a Gilbert multiplier. Each case uses the following update law $\dot{W}=-\eta \cdot \frac{\partial E}{\partial W}-\alpha \cdot W$, where $N E T^{(i)}=\tanh \left(W^{(i)}\right) \cdot \tanh \left(X^{(i-1)}\right)$
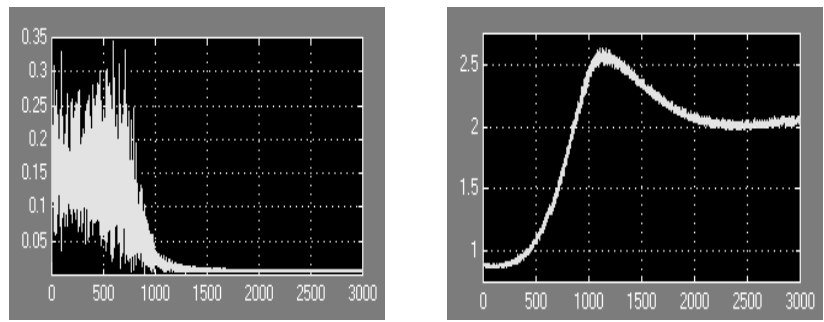

Figure 4: Training Error and convergence for the modified BP Algorithm with non-linear multiplier 
The update laws are

$$
\begin{aligned}
\dot{W}^{(3)}= & \eta \cdot \tanh (D-Y) \cdot \tanh \left(X^{(3)}\right)-\alpha \cdot W^{(3)} \\
\dot{W}^{(2)}= & \eta \cdot \tanh \left(\tanh \left(\psi^{\prime}\left(N E T^{(2)}\right)\right) \cdot \tanh (\tanh (D-Y) \cdot\right. \\
& \left.\left.\tanh \left(W^{(3)}\right)\right)\right) \cdot \tanh \left(X^{(2)}\right)-\alpha \cdot W^{(2)} \\
\dot{W}^{(1)}= & \eta \cdot \tanh \left(\tanh \left(\psi^{\prime}\left(N E T^{(1)}\right)\right) \cdot\right. \\
& \tanh \left(\operatorname { t a n h } \left(\tanh \left(\psi^{\prime}\left(N E T^{(2)}\right)\right) \cdot \tanh (\tanh (D-Y)\right.\right. \\
& \left.\left.\left.\left.\cdot \tanh \left(W^{(3)}\right)\right)\right) \cdot \tanh \left(W^{(2)}\right)\right)\right) \cdot \tanh \left(X^{(1)}\right)-\alpha \cdot W^{(1)}
\end{aligned}
$$

3.3 The Modified BP algorithm with nonlinear multipliers and the removal of the derivative function in all the hidden layers.

The update laws are

$$
\begin{aligned}
& \dot{W}^{(3)}=\eta \cdot \tanh (D-Y) \cdot \tanh \left(X^{(3)}\right)-\alpha \cdot W^{(3)} \\
& \dot{W}^{(2)}=\eta \cdot \tanh \left(\tanh (D-Y) \cdot \tanh \left(W^{(3)}\right)\right) \cdot \tanh \left(X^{(2)}\right) \\
& \dot{W}^{(1)}=\eta \cdot \alpha \cdot W^{(2)} \\
& \cdot \tanh \left(\tanh \left(\tanh (D-Y) \cdot \tanh \left(W^{(3)}\right)\right) \cdot \tanh \left(W^{(2)}\right)\right)
\end{aligned}
$$
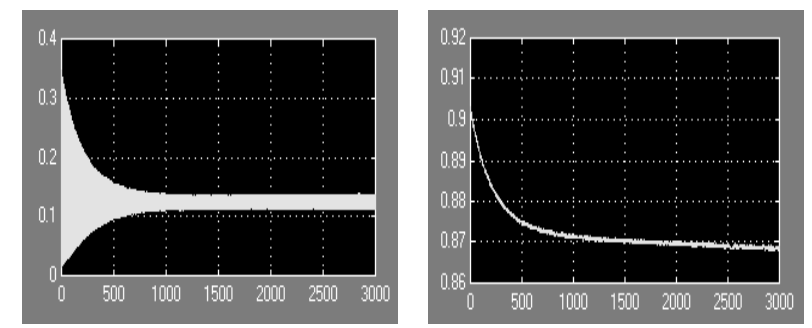

Figure 5: Training Error and convergence for modified BP Algorithm with removal of derivative function in all hiden layers

Presented in fig. 6 are the input/output waveforms for the network trained using the above final update rule

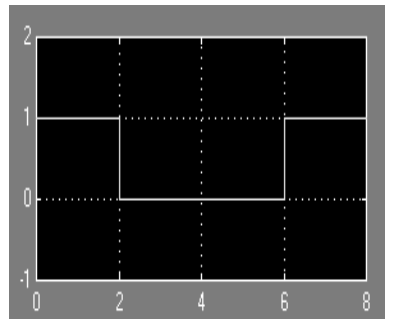

Test Input 1

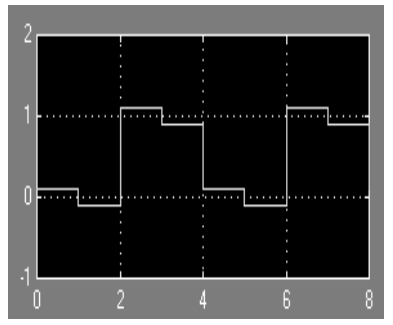

Test Input 2

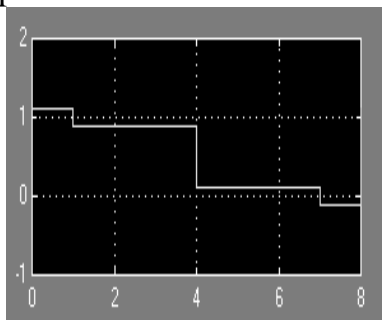

Test Ouput

Figure 6: Input /Output waveforms for the final network

\subsection{Summary of the High-level Simulation Results}

From the high-level Matlab/Simulink simulations, one can draw the following conclusions:

1) In replacing the ideal linear multiplier model by the realistic nonlinear multiplier model, the neural network converges.

2) Removing the derivative function of the second hidden layer, the neural network could still converge. In fact, this can be easily verified mathematically.

3) When the derivative function in all hidden layers are removed, the neural network could converge, but in this case the training error is not zero but attains a small constant mean value. The update law derived in this case is still a gradient type law [7].

For all presented simulation results, we used the same set of initial conditions for all the models i.e all initial conditions at zero. In general for different nonlinear systems, the same initial conditions may bring different training result. An initial weight set which results in a good training result for one nonlinear system will not necessarily ensure that it will result in a good training result for another nonlinear system.

\section{Conceptual Chip Design}

Presented below is a higher block level design of the chip. For more details see [1,9]

\subsection{The Synaptic Cell:}

The initial and highest level design of each neural cell shows the central idea of the processing network and the learning network (Fig. 7).

The processing stage is composed of 16 neurons built using vector multipliers and a sigmoid function. The multipliers use as operands an input vector and a weight vector. The input is common to all processing units, and the weights belong to each neuron. The scalar product is then applied to the non-linear function, resulting in the output of a neuron.

The learning stage works in a similar manner as the processing stage, but using different sources for the product. It receives the signals from the next stage, creates a new signal to be sent to any previous cell, and updates the weights according to the update law. Both the stages of learning and the processing networks are merged locally. To accomplish this, it was necessary to decompose the 17D multipliers that constituted each network node into a set of 1-D multipliers.

On-chip memory is designed as local digital memory. It is therefore necessary to add a stage where the current analog 


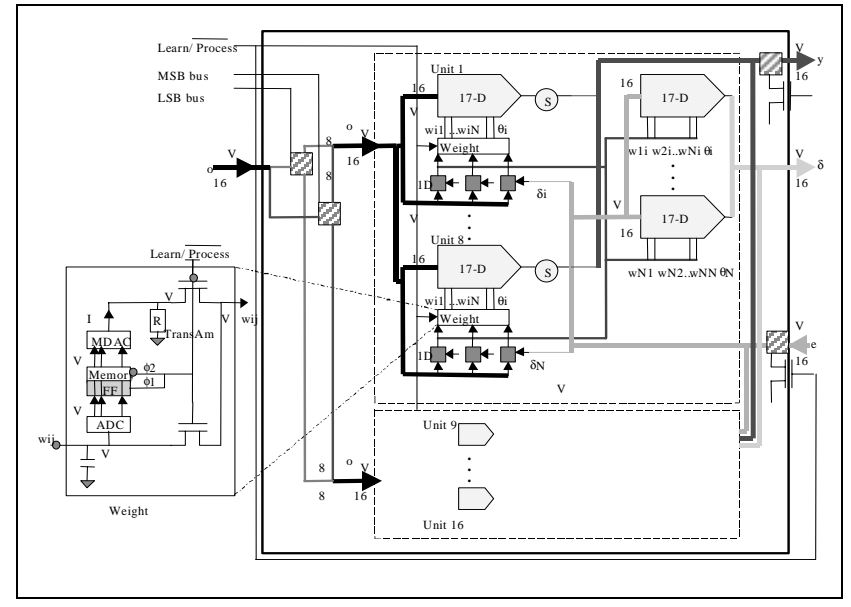

Figure 7: Architectural Block Diagram - Synapse

value of the weight is converted into a digital value using an ADC, and then converted back using a DAC. The memory is built by using 5 data flip-flops. The update law, however, uses a capacitor (see Fig. 7) and 1-dimension (1D) multipliers. These multipliers are also used in each neuron, to form the 17-dimension (17-D) multipliers.

To optimize the number of ADCs required for the conversion of the weight and still achieve good performance, an array of ADCs was designed away from the neural network. With this new configuration one ADC could be shared by the whole row of weights, reducing the number of ADCs to $\sqrt{n}$. This design uses multiplexers, decoders, control logic for the store mode and the need of a clocked input to drive this logic. This clock also regulates the ADC operation, as it is designed to be successive approximations type. Please note that having a clock in this section does not imply that the neural network stops being asynchronous. For more detailed review of the chip architecture, kindly refer to $[1,8,9]$.

\section{Design and Layout of Components}

There are a number of custom designed components for this chip. All the component circuits were designed on StarHspice using BSIM Level-49 models supplied by SRC/UMC. Avant's software was used for the schematic entry and waveform viewing. Initial layout of the subcircuits was carried out in Tanner Tools but the verification and LVS were performed using Cadence Tools. In this paper we restrict ourselves to presenting results on the more vital components of the chip. This includes a Gilbert multiplier, a wide transconductance amplifier and a comparator in this paper. In addition, demonstrative simulations for $\mathrm{ADC}$ and the vector multiplier are also provided.

\subsection{Gilbert Multiplier}

To implement the multiplication in the analog domain, a Gilbert multiplier cell has been employed. The circuit diagram of the modified Gilbert multiplier is shown in fig. 8. Assume that all transistors in fig. 8 are in saturation region, and are matched so that the trans-conductance parameters satisfy the equations

$\beta_{\mathrm{N}}=\beta_{\mathrm{M} 1}=\beta_{\mathrm{M} 2}$ and $\beta_{\mathrm{P}}=\beta_{8}=\beta_{9}=\beta_{10}=\beta_{11}$

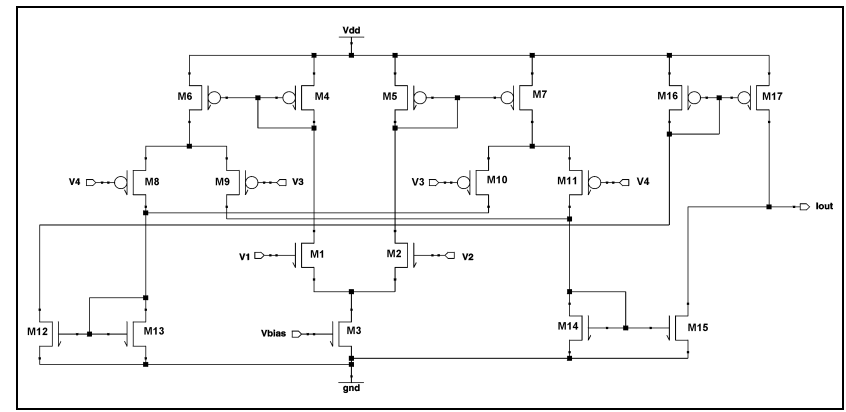

Figure 8: Gilbert Multiplier - Schematics

The output current then is the difference between $\mathrm{I}_{\mathrm{D}(\mathrm{M} 13)}$ and $\mathrm{I}_{\mathrm{D}(\mathrm{M} 14)}$ since the current $\mathrm{I}_{\mathrm{S}(\mathrm{M} 16)}$ and $\mathrm{I}_{\mathrm{S}(\mathrm{M} 17)}$ are reflected by the current mirrors.

Defining the output currents

$I_{+}=I_{S(M 8)}+I_{S(M 10)}$

$I_{-}=I_{S(M 9)}+I_{S(M 11)}$

it can be readily shown that the ideal characteristics of differential output current $I_{D I F F}=I_{+}-I_{-}$is given by $I_{\text {DIFF }}=\sqrt{\beta_{p} \beta_{N}}\left(\left(V_{3}-V_{4}\right)\left(V_{1}-V_{2}\right)\right)$

The modified Gilbert multiplier takes the difference between two voltages $\left(\mathrm{V}_{3}-\mathrm{V}_{4}\right)$ and multiplies that difference by a difference of two other voltages (V1-V2). In the small signal range, the characteristic curve is approximately linear, with all four inputs carrying multiplication information. For the large signal range, the multiplier is non-linear but does not cause any instability.

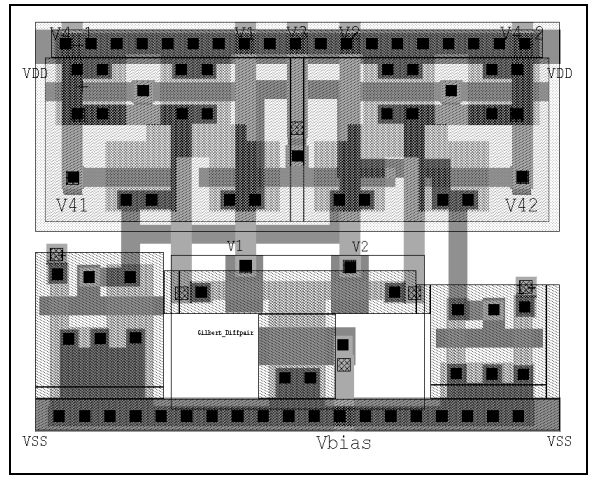

Figure 9: Gilbert Multiplier - Layout

The multiplier layout is shown in fig. 9, while the HSPICE simulated DC characteristics are shown in fig. 10 and shows four quadrant multiplication 


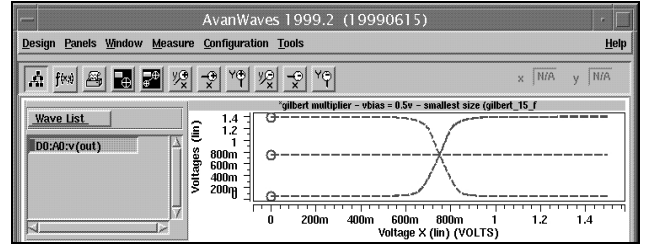

Figure 10: Gilbert Multiplier - DC Characteritics

\subsection{Wide Transconductance Amplifier}

The wide-transconductance amplifier is used in multiple modes in this chip. It is used as the sigmoid non-linearity at the end of each row, and as a buffer for in-chip signal buffering.

For the transconductance amplifier, the differential-in, differential-out transconductance is given by the equation

$$
g_{\text {md }}=\frac{\partial I_{\text {out }}}{\partial V_{I D}}=\sqrt{\beta \mathrm{I}_{\mathrm{D} 3}}=\sqrt{\frac{\mathrm{K}_{1} \mathrm{I}_{\mathrm{D} 3} \mathrm{~W}_{1}}{\mathrm{~L}_{1}}} \quad\left(\text { at } V_{I D}=0\right)
$$

Wide transconductance amplifier was preferred over the simple transconductance amplifier for its better characteristics against

- transistor size/current mismatch and hence commonmode gain

- input common-mode voltage range

- better input/output voltage swing

The designed amplifier was achieved by adding two extra current mirrors to the simple transconductance amplifier. By reflecting the currents of $\mathrm{Ml}$ and $\mathrm{M} 2$ to the upper current mirrors, the output current is just the difference between $I_{1}$ and $I_{2}$, with the advantage that both input and output voltages can run almost up to $\mathrm{V}_{\mathrm{dd}}$ and almost down to $\mathrm{V}_{\mathrm{ss}}$, without affecting the operation of the circuit.

The output current, $I_{\text {out }}$, in the schematic (fig. 11), is converted to a voltage value using a 2-CMOS active load (not shown in the schematics). The complete layout is shown in fig. 12 with the simulated transfer characteristics shown in fig. 13.

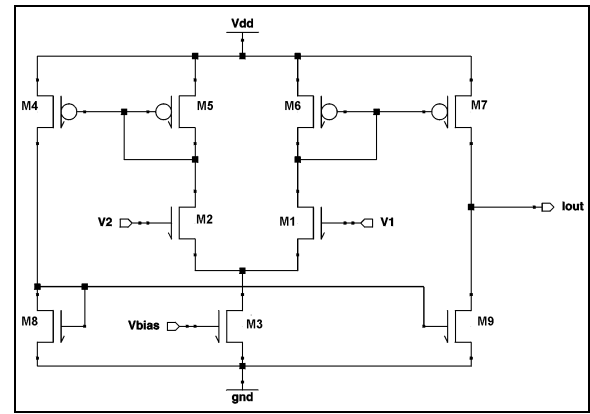

Figure 11: Wide Transamp - Schematics

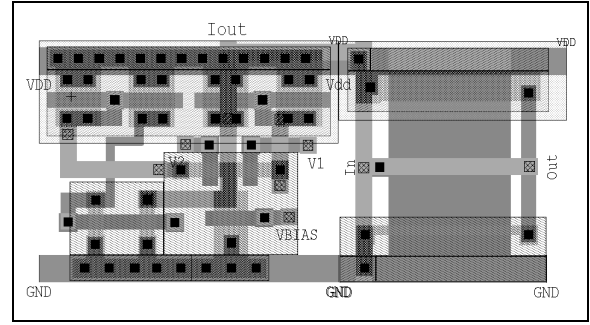

Figure 12: Wide Transamp - Layout

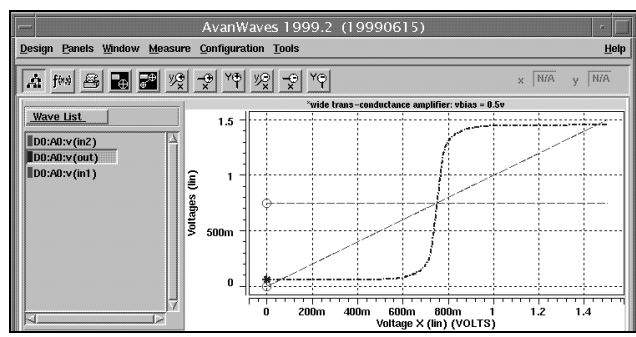

Figure 13: Wide Transamp - DC Characteristics

\subsection{Comparator}

The comparator is used in ADC to compare the Multiplying DAC output voltage with the actual input voltage that is to be converted. The input stage of the comparator is a differential amplifier and the next stage is a decision circuit. The last stage is nothing but an inverter used as a thresholding/polarity correction circuit. The schematic is shown in fig. 14, the layout in fig. 15 and the characteristics waveforms are shown in fig. 16.

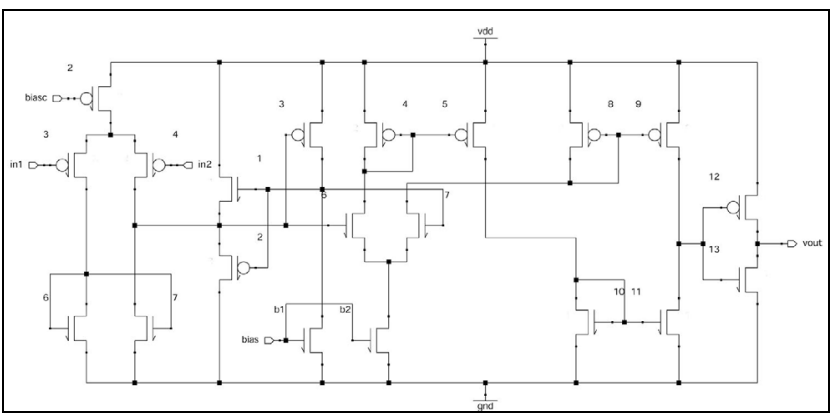

Figure 14: Threshold Comparator- Schematics

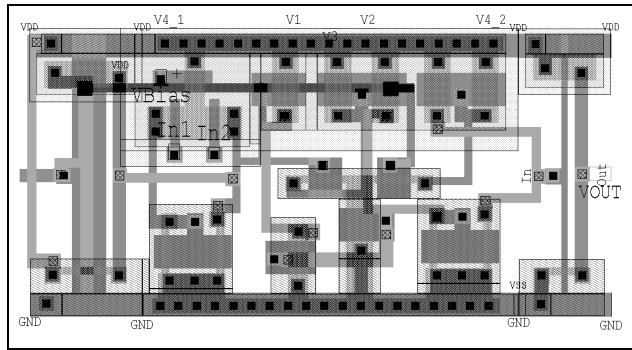

Figure 15: Threshold Comparator- Layout 


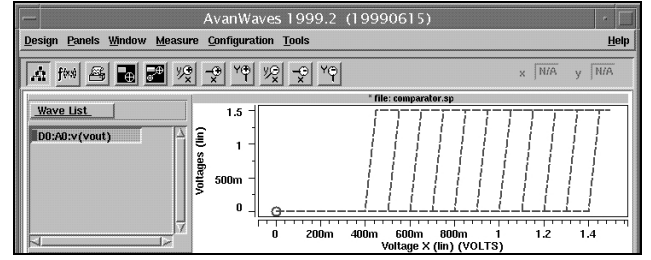

Figure 16: Threshold Comparator- Characteristics

\subsection{ADC Operation}

The simulation results below illustrate a simulation of conversion of an analog voltage of $0.85 \mathrm{~V}$ to its digital equivalent by the successive approximation ADC internal to the chip. In this simulation the conversion process takes approximately $3 \mu \mathrm{s}$ to convert, implying that all the converged analog weights will be converted to their digital equivalent in approximately $3 \mu \mathrm{s} \times 17=51 \mu \mathrm{s}$.

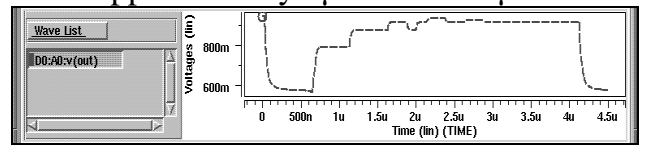

Figure 17: ADC Convergence Characteristics

\subsection{Vector Multiplications}

Presented below are the transient characteristics of multiplication of two time domain sinusoids. The third waveform presents the result, which is the cascaded result of the current output of seventeen multipliers collected in current busbars and converted to the voltage domain at the end using a 4-CMOS active load.

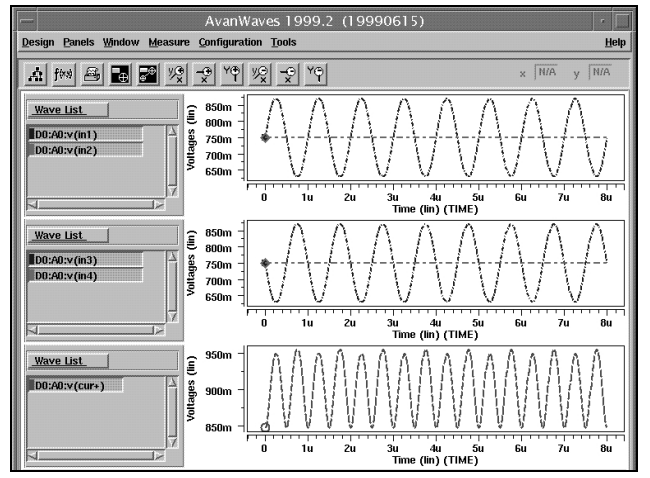

Figure 18: Vector Signal Multiplication- Transient

\section{Overall Chip Layout \& Interconnects}

The figure below shows the array structure of the implemented chip, best possible fit of the building sub-cells were tried in order to achieve a highly dense building block structure. A hierarchical routing assignment was made for the available 6 metal layers to achieve the required dense connectivity. For more details see [1].

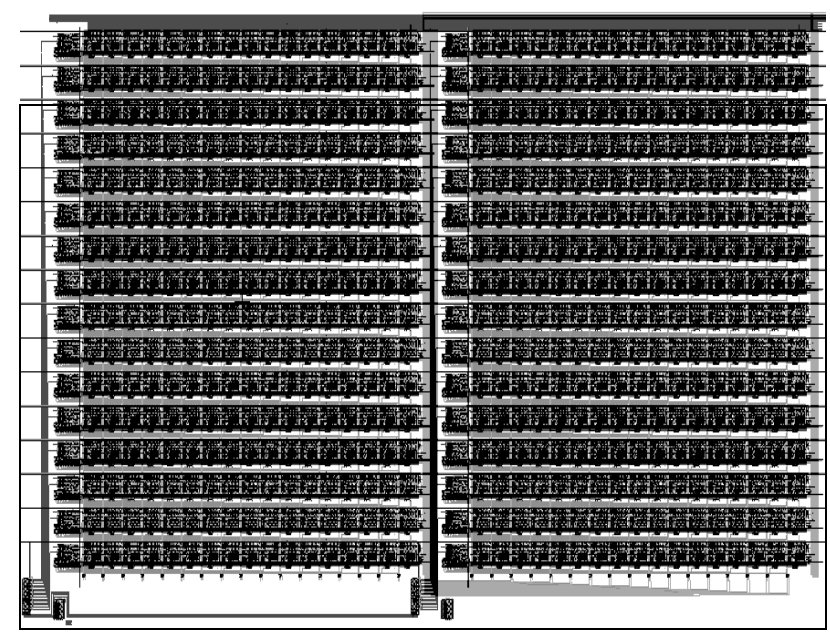

Figure 19: Layout for the Building Block

\section{References}

[1] Khurram Waheed and Fathi M. Salam, "A MixedMode Design for a Self-programming Chip For real-time estimation, prediction, and control"; Proc. Of $43^{\text {rd }}$ IEEE Midwest Symposium on Circuits and Systems, Aug 8-11, 2000, pp. 810-813

[2] Gert Cauwenberghs and M. Bayoumi, (editors) Learning on Silicon, adaptive VLSI neural systems, Kluwer Academic Publishers, July 1999.

[3] F. M. Salam, H-J. Oh, "Design of a Temporal Learning Chip for Signal Generation and Classification," Analog Integrated Circuits and Signal Processing, an international journal, Kluwer Academic Publishers, Vol. 18, No. 2/3, February 1999, pp. 229-242.

[4] M. Ahmadi and F. Salam, Special Issue on Digital and Analog Arrays, International Journal on Circuits, Systems, and Computers, October/December 1998 (Issue published in December 1999).

[5] F. M. Salam, M. R. Choi."An All-MOS Analog Feedforward Neural Circuit with Learning," IEEE Int' Symp. on Circuits and Systems (ISCAS), May 1990, pp. 2508-2511.

[6] MSU Team, Copper IC Design Challenge, Phase I Report, January 2000.

[7] MSU Team, Copper IC Design Challenge, Phase II Report, August 2000.

[8] Website:http://www.egr.msu.edu/annweb/cu_contest/ 